\title{
Global description of bottomonium suppression in proton-nucleus and nucleus-nucleus collisions at LHC energies
}

\author{
Elena G. Ferreiro* \\ Departamento de Física de Partículas and IGFAE, Universidade de Santiago de Compostela, \\ 15782 Santiago de Compostela, Spain \\ Laboratoire Leprince-Ringuet, Ecole polytechnique, CNRS/IN2P3, Université Paris-Saclay, \\ F-91128 Palaiseau, France \\ E-mail: elena@fpaxpl.usc.es
}

\begin{abstract}
We show that we can reach a global and coherent description of bottomonium suppression in both proton-nucleus and nucleus-nucleus collisions at the LHC energies. The measured relative suppression of the excited bottomonium states as compared to their ground state allows us to constrain the scattering cross sections between the bottomonia and the comovers created during the collisions. Our result hints at a similar energy distribution of these comovers in the environment created by proton-nucleus and nucleus-nucleus collisions. Along the way of our study, we also update our knowledge for the bottomonium feed-down pattern and our comprehension on the modification of the nuclear parton distribution functions in proton-nucleus collisions. We present our results for $p \mathrm{~Pb}$ collisions at 5.02 and $8.16 \mathrm{TeV}$ and for $\mathrm{PbPb}$ collisions at 2.76 and 5.02 $\mathrm{TeV}$.
\end{abstract}

XXVI International Workshop on Deep-Inelastic Scattering and Related Subjects (DIS2018)

16-20 April 2018

Kobe, Japan

${ }^{*}$ Speaker. 


\section{Introduction}

The idea of quarkonium sequential suppression as a signature of the formation of a hot quarkgluon plasma (QGP) [1] - different quarkonium states would melt sequentially at different temperatures within a deconfined medium-, was supported by the relative suppression of bottomonium observed by CMS Collaboration in lead-lead collisions [2, 3]: the excited $\Upsilon(2 S)$ and $\Upsilon(3 S)$ states were suffering more suppression than the ground $\Upsilon(1 \mathrm{~S})$ state.

However, a couple of years later, the same Collaboration found a similar effect [4] -although of different magnitude- in proton-lead collisions, where no QGP formation was expected. Moreover, a relative suppression of charmonium was equally found by PHENIX [5] and ALICE [6] Collaborations, also in asymmetric proton(deuteron)-nucleus collisions. While at lower-SPS-energies the relative suppression could be attributed to the interaction of the different quarkonium states -with different break-up cross sections- with the remnants of the colliding nucleus, at higher energies the quarkonium formation time will be boosted. This implies that in this case the $Q \bar{Q}$ pair does not have the time to evolve into any physical state when it escapes the nuclear matter. Thus, one cannot appeal to this mechanism to justify the observed difference, neither can one invoke initial-state effects -as the modification of the nuclear parton distribution functions (nPDFs)- which should have a similar impact on the different states.

A natural explanation could be a final-state effect acting over sufficiently long time. According to the data, this effect should be stronger in the nucleus-going direction, and it should increase with centrality. Already in the late 90's, an effect of this type, based on the interaction of the quarkonium with the comoving medium -i.e. made of particles with similar rapidities and whose density is directly connected to the particle multiplicity- was introduced. Here, we propose to explain the relative suppression of bottomonia in both $p \mathrm{~Pb}$ and $\mathrm{PbPb}$ LHC collisions by this interaction.

\section{The improved comover interaction model (ICIM)}

Within this model $[7,8,9]$, quarkonia are suppressed due to scatterings with the comoving particles. The evolution equation that governs the density of bottomonium at a given transverse coordinate $s$, impact parameter $b$ and rapidity $y, \rho^{\Upsilon}(b, s, y)$, follows the expression

$$
\tau \frac{\mathrm{d} \rho^{\Upsilon}}{\mathrm{d} \tau}(b, s, y)=-\left\langle\sigma^{c o-\Upsilon}\right\rangle \rho^{c o}(b, s, y) \rho^{\Upsilon}(b, s, y),
$$

where $\left\langle\sigma^{\mathrm{co}-\Upsilon}\right\rangle$ corresponds to the interaction cross section of bottomonium with the comoving medium of transverse density $\rho^{c o}(b, s, y)$. It depends essentially on 2 parameters, applicable to the entire bottomonium family. It reads

$$
\left\langle\sigma^{\mathrm{co}-\Upsilon}\right\rangle\left(T_{\mathrm{eff}}, n\right)=\frac{\int_{0}^{\infty} d E^{\mathrm{co}} \mathscr{P}\left(E^{\mathrm{co}} ; T_{\mathrm{eff}}\right) \sigma^{\mathrm{co}-\Upsilon}\left(E^{\mathrm{co}}\right)}{\int_{0}^{\infty} d E^{\mathrm{co}} \mathscr{P}\left(E^{\mathrm{co}} ; T_{\mathrm{eff}}\right)}, \text { where } \mathscr{P}\left(E^{\mathrm{co}} ; T_{\mathrm{eff}}\right) \propto \frac{1}{e^{E^{\mathrm{co}} / T_{\mathrm{eff}}}-1}
$$

corresponds to the Bose-Einstein energy distribution of the comovers, which introduces our first parameter, the effective temperature of these comovers $T_{\text {eff }}$. 
Our parametrisation of the energy dependence for the cross section $\sigma^{\mathrm{co}-\Upsilon}\left(E^{\mathrm{co}}\right)$ interpolates from $\sigma^{\mathrm{co}-\Upsilon}\left(E^{\mathrm{co}}=E_{\mathrm{thr}}^{\Upsilon}\right)=0$ at threshold up to the geometrical cross section, $\sigma^{\mathrm{co}-\Upsilon}\left(E^{\mathrm{co}} \gg E_{\mathrm{thr}}^{\mathrm{r}}\right)=$ $\sigma_{\text {geo }}^{\Upsilon}$, where $\sigma_{\text {geo }}^{\Upsilon} \simeq \pi r_{\Upsilon}^{2}$, being $r_{\Upsilon}$ the bottomonium Bohr radius. It reads

$$
\sigma^{\mathrm{co}-\mathrm{r}}\left(E^{\mathrm{co}}\right)=\sigma_{\mathrm{geo}}^{\mathrm{r}}\left(1-\frac{E_{\mathrm{thr}}^{\mathrm{r}}}{E^{\mathrm{co}}}\right)^{n}
$$

where $E_{\mathrm{thr}}^{\Upsilon}=M_{\Upsilon}+m_{\mathrm{co}}-2 M_{B}$ is the threshold energy to break the bottomonium bound state and $E^{\mathrm{co}}=\sqrt{p^{2}+m_{\mathrm{co}}^{2}}$ is the energy of the comover in the quarkonium rest frame. The second parameter of our modeling, $n$, characterises how quickly the cross section approaches the geometrical cross section. We will let $n$ varying from 0.5 to 2 .

In order to proceed with the fit, it is mandatory to take into account the feed-down contributions. The feed-down fractions [10] for the $\Upsilon(1 \mathrm{~S})$ can be estimated as: $70 \%$ of direct $\Upsilon(1 \mathrm{~S}), 8 \%$ from $\Upsilon(2 S)$ decay, $1 \%$ from $\Upsilon(3 S), 15 \%$ from $\chi_{\mathrm{B} 1}, 5 \%$ from $\chi_{\mathrm{B} 2}$ and $1 \%$ from $\chi_{\mathrm{B} 3}$, while for the $\Upsilon(2 \mathrm{~S})$ the different contributions would be: $63 \%$ direct $\Upsilon(2 S), 4 \%$ of $\Upsilon(3 S), 30 \%$ of $\chi_{\mathrm{B} 2}$ and $3 \%$ of $\chi_{\mathrm{B} 3}$. For the $\Upsilon(3 \mathrm{~S}), 40 \%$ of the contribution will come from decays of $\chi_{\mathrm{B} 3}$.

We have performed our fit of $T_{\text {eff }}$ for different values of $n$ with both gluon $\left(m_{\mathrm{co}}=0\right)$ or pion $\left(m_{\mathrm{co}}=0.140 \mathrm{GeV}\right)$ comovers. For $p$ Pb collisions, we have used the CMS [4] and ATLAS [11] data at $5.02 \mathrm{TeV}$ on relative nuclear suppression factors. For $\mathrm{PbPb}$ collisions, we have used the CMS data at 2.76 TeV [3] and at 5.02 TeV [12], also on relative nuclear suppression factors. Our results are shown in Fig. 1. All the combinations yield to $T_{\mathrm{eff}} \mathrm{S}$ in the range 200 to $300 \mathrm{MeV}$ for

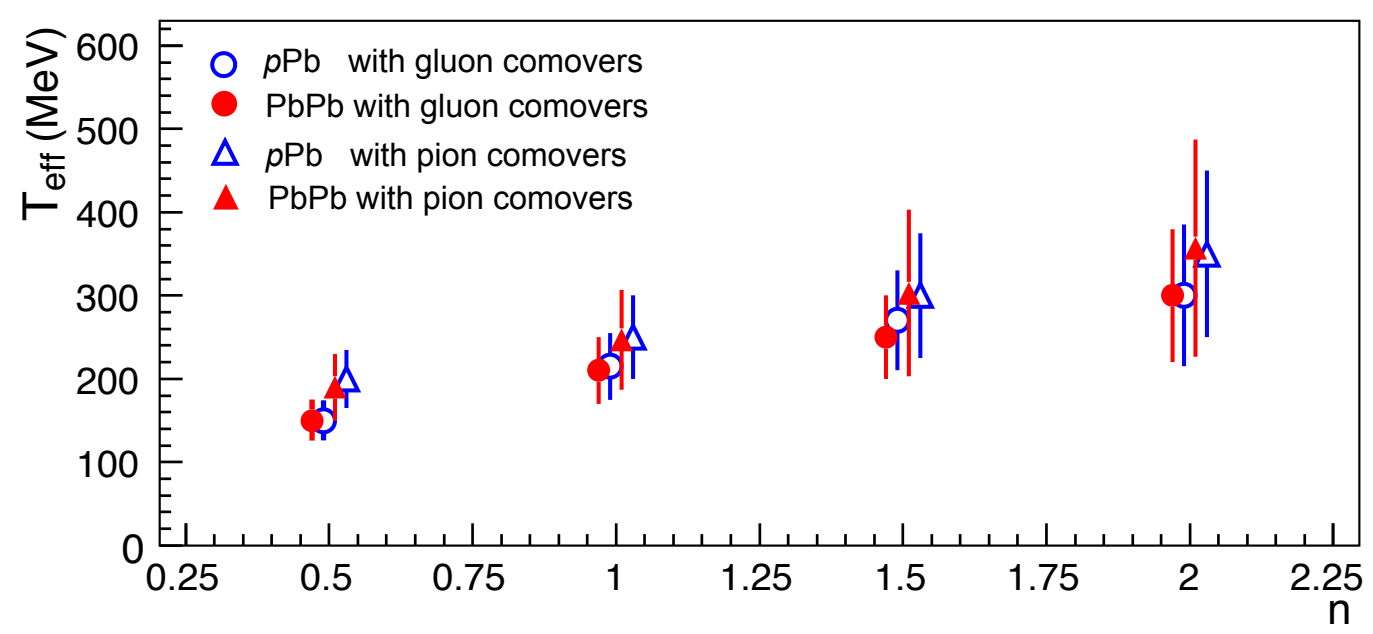

Figure 1: Fitted $T_{\text {eff }}$ considering pion (triangles) or gluon (circles) comovers from our fits to $p \mathrm{~Pb}$ (empty blue) and $\mathrm{PbPb}$ (filled red) data for different $n$ from 0.5 to 2 . [The points have been horizontally shifted for readability.]

our assumed range for $n$. In what follows, our results will be shown for $n=1$ and $T_{\text {eff }}=250 \pm 50$ $\mathrm{MeV}$. Choosing different couples of $n$ and $T_{\text {eff }}$ yield to very similar results since the variation of $n$ is compensated by that of $T_{\text {eff. }}$.

\section{Results}

Our results for the relative nuclear modification factors in $p \mathrm{~Pb}$ collisions at $5.02 \mathrm{TeV}$ are presented in Table 1 together with the CMS [4] and ATLAS [11] experimental data. 


\begin{tabular}{lcc}
\hline \hline & ICIM fit & Experimental values \\
\hline & $-1.93<y<1.93$ & CMS data \\
$\Upsilon(2 S) / \Upsilon(1 S)$ & $0.91 \pm 0.03$ & $0.83 \pm 0.05$ (stat.) \pm 0.05 (syst.) \\
$\Upsilon(3 S) / \Upsilon(1 S)$ & $0.72 \pm 0.02$ & $0.71 \pm 0.08$ (stat.) \pm 0.09 (syst.) \\
& $-2.0<y<1.5$ & ATLAS data \\
$\Upsilon(2 S) / \Upsilon(1 S)$ & $0.90 \pm 0.03$ & $0.76 \pm 0.07$ (stat.) \pm 0.05 (syst.) \\
$\Upsilon(3 S) / \Upsilon(1 S)$ & $0.71 \pm 0.02$ & $0.64 \pm 0.14$ (stat.) \pm 0.06 (syst.) \\
\hline \hline
\end{tabular}

Table 1: $R_{p P b}^{\Upsilon(\mathrm{nS}) / \Upsilon(1 \mathrm{~S})}$ at $5.02 \mathrm{TeV}$

Fig. 2 shows our results on the centrality dependence of the relative suppression of $\Upsilon(\mathrm{nS})$ at 2.76 and $5.02 \mathrm{TeV}$ for $\mathrm{PbPb}$ collisions compared to the CMS data $[3,12]$.

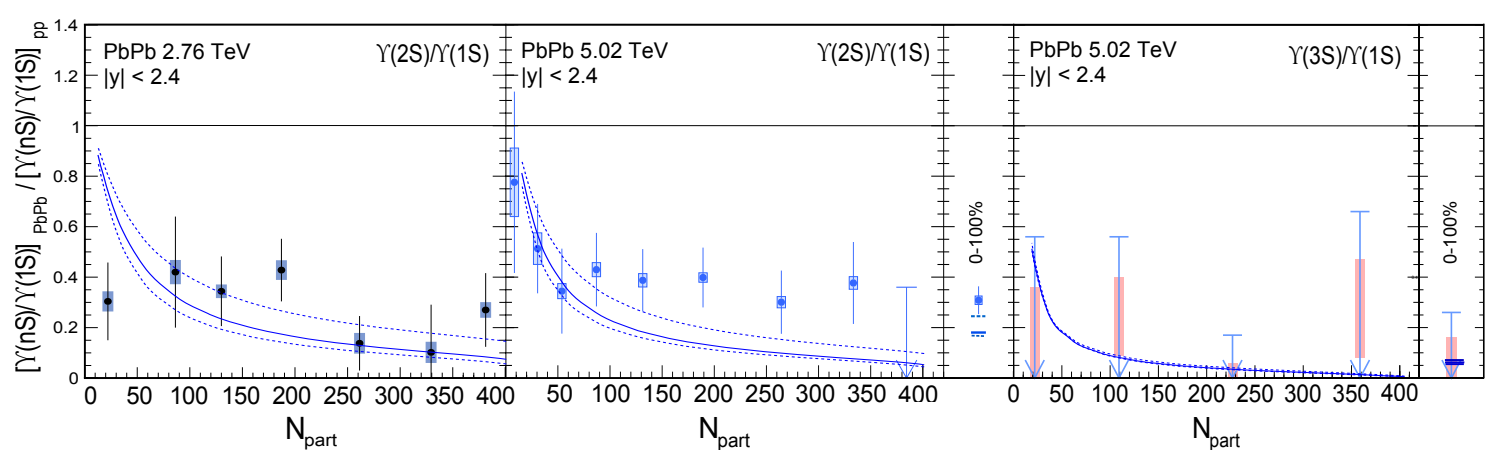

Figure 2: The double ratio $\left(R_{P b P b}^{\Upsilon(\mathrm{nS}) / \Upsilon(1 \mathrm{~S})}\right)$ for $\Upsilon(2 \mathrm{~S})$ over $\Upsilon(1 \mathrm{~S})$ at 2.76 and $5.02 \mathrm{TeV}$ and $\Upsilon(3 \mathrm{~S})$ over $\Upsilon(1 \mathrm{~S})$ at 5.02 TeV as a function of $N_{\text {part }}$ obtained from the ICIM and compared to the CMS data at $2.76 \mathrm{TeV}$ [3] and 5.02 TeV [12]. The dashed line depicts the uncertainty from the fit of $\sigma^{c o-\Upsilon}$.

Once the parameters of our approach have been fixed with the relative suppression measurements, we can address the absolute suppression of each state. In this case, other nuclear effects, which cancel in the double ratio of the excited-to-ground state suppression, do not cancel anymore. At LHC energies, the main one is the nuclear modification of parton distribution functions. It is easily accounted for by using available global nPDF fits with uncertainties.

In Fig. 3, we show $R_{p \mathrm{~Pb}}^{\Upsilon(1 S)}$ vs rapidity at $\sqrt{s}=5.02 \mathrm{TeV}$ compared to the available experimental data $[11,13,14]$ from ATLAS, LHCb and ALICE. An overall good agreement is obtained. Going to $\mathrm{PbPb}$ collisions, our results for the $3 \Upsilon$ states at 2.76 and $5.02 \mathrm{TeV}$ are also shown in Fig. 3 together with the CMS data $[15,16]$. A good agreement is obtained in the 3 cases with the same parameters used to reproduce the relative suppression. The nCTEQ15 shadowing [17] has been taken into account.

To finish, in Fig. 4, our predictions for $p \mathrm{~Pb}$ collisions at $8.16 \mathrm{TeV}$ are shown using two different parametrisations for the shadowing, nCTEQ15 [17] and EPS09LO [18]. Our results agree with the recent data obtained by LHCb Collaboration [19]. 

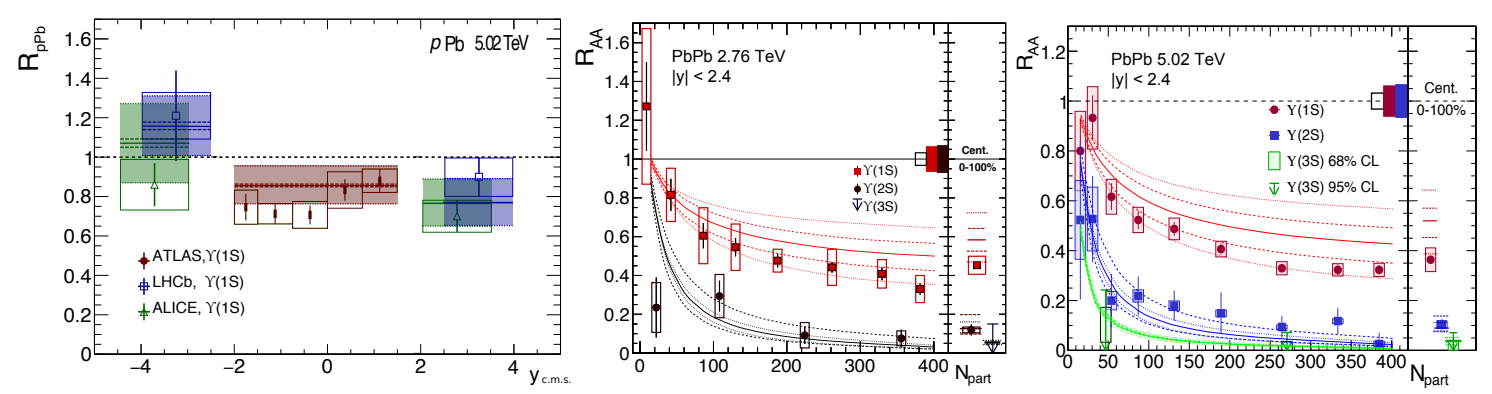

Figure 3: $R_{p \mathrm{~Pb}}^{\mathrm{\Upsilon}(1 S)}$ vs rapidity (right) at $5.02 \mathrm{TeV}$ compared to the LHC data $[13,14,11]$ and $R_{P b P b}^{\Upsilon(n S)}$ vs $N_{\text {part }}$ (left) compared to the CMS data at $2.76 \mathrm{TeV}$ [15] and $5.02 \mathrm{TeV}$ [16]. The uncertainty from the fit of $\sigma^{c o-\Upsilon}$ (dashed line) and from the nCTEQ15 shadowing (dotted line) are shown separately.
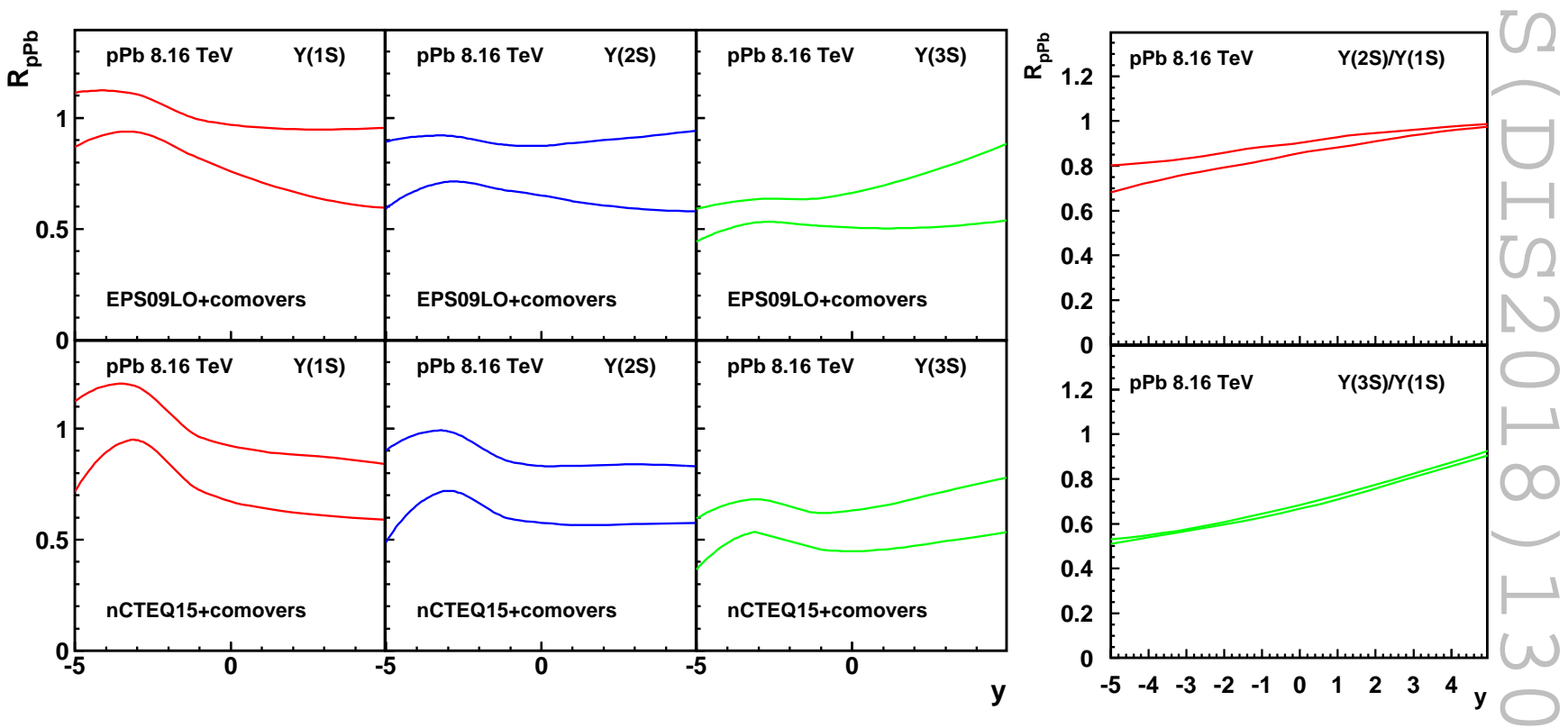

Figure 4: $\quad R_{p \mathrm{~Pb}}^{\Upsilon(n S)}$ vs rapidity at $8.16 \mathrm{TeV}$. Two different shadowing parametrisations (nCTEQ15 and EPS09LO) have been taken into account.

\section{Conclusions}

The relative suppression of the excited bottomonium states as compared to their ground state can be explained by the interaction with comovers. We propose a generic formula for all the quarkonia states and suggest a connection with the energy distribution of the comovers, thus with an effective local temperature. This leads to a consistent magnitude for the $\Upsilon$ suppression in both $p \mathrm{~Pb}$ and $\mathrm{PbPb}$ collisions when combined with shadowing.

The nature of comovers could correspond to 2 different scenarios. If the comovers are identified with partons, the ICIM provides an effective modelling of bottomonium dissociation in the QGP. If the comovers are identified with hadrons both in $p A$ and $A A$ collisions, this would imply that the $\Upsilon$ s remain unaffected by the presence of a possible QGP. Obviously, more complex -and intermediate- scenarios could be proposed, where the suppression takes place both with partons and hadrons. 


\section{References}

[1] T. Matsui and H. Satz, J/ $\psi$ Suppression by Quark-Gluon Plasma Formation, Phys. Lett. B178 (1986) 416-422.

[2] CMS collaboration, S. Chatrchyan et al., Indications of suppression of excited $\Upsilon$ states in $\mathrm{PbPb}$ collisions at $\sqrt{S_{N N}}=2.76$ TeV, Phys. Rev. Lett. 107 (2011) 052302, [1105.4894].

[3] CMS collaboration, S. Chatrchyan et al., Observation of sequential Upsilon suppression in PbPb collisions, Phys. Rev. Lett. 109 (2012) 222301, [1208 . 2826].

[4] CMS collaboration, S. Chatrchyan et al., Event activity dependence of $Y(n S)$ production in $\sqrt{s_{N N}}=5.02 \mathrm{TeV} \mathrm{pPb}$ and $\sqrt{s}=2.76 \mathrm{TeV}$ pp collisions, JHEP 04 (2014) 103, [1312 . 6300].

[5] PHENIX collaboration, A. Adare et al., Nuclear Modification of $\psi^{\prime}, \chi_{c}$ and $J / \psi$ Production in $d+A u$ Collisions at $\sqrt{s_{N N}}=200$ GeV, Phys. Rev. Lett. 111 (2013) 202301, [1305. 5516].

[6] ALICE collaboration, B. B. Abelev et al., Suppression of $\psi(2 S)$ production in $p$-Pb collisions at $\sqrt{s_{\mathrm{NN}}}=5.02 \mathrm{TeV}$, JHEP 12 (2014) 073, [1405.3796].

[7] E. G. Ferreiro, Charmonium dissociation and recombination at LHC: Revisiting comovers, Phys. Lett. B731 (2014) 57-63, [1210.3209].

[8] E. G. Ferreiro, Excited charmonium suppression in proton-nucleus collisions as a consequence of comovers, Phys. Lett. B749 (2015) 98-103, [1411.0549].

[9] E. G. Ferreiro and J.-P. Lansberg, Is bottomonium suppression in proton-nucleus and nucleus-nucleus collisions at LHC energies due to the same effects?, 1804.04474.

[10] A. Andronic et al., Heavy-flavour and quarkonium production in the LHC era: from proton-proton to heavy-ion collisions, Eur. Phys. J. C76 (2016) 107, [1506.03981].

[11] ATLAS collaboration, M. Aaboud et al., Measurement of quarkonium production in proton-lead and proton-proton collisions at 5.02 TeV with the ATLAS detector, Eur. Phys. J. C78 (2018) 171.

[12] CMS collaboration, A. M. Sirunyan et al., Suppression of Excited $\Upsilon$ States Relative to the Ground State in Pb-Pb Collisions at $\sqrt{s_{\mathrm{NN}}}=5.02$ TeV, Phys. Rev. Lett. 120 (2018) 142301, [1706. 05984 ].

[13] LHCb collaboration, R. Aaij et al., Study of $\Upsilon$ production and cold nuclear matter effects in $p P b$ collisions at $\sqrt{s_{N N}}=5 \mathrm{TeV}$, JHEP 07 (2014) 094, [1 405 . 5152].

[14] ALICE collaboration, B. B. Abelev et al., Production of inclusive $\Upsilon(1 S)$ and $\Upsilon(2 S)$ in $p$-Pb collisions at $\sqrt{\mathbf{s}_{\mathrm{NN}}}=\mathbf{5 . 0 2}$ TeV, Phys. Lett. B740 (2015) 105-117, [1410.2234].

[15] CMS collaboration, V. Khachatryan et al., Suppression of $\Upsilon(1 S), \Upsilon(2 S)$ and $\Upsilon(3 S)$ production in PbPb collisions at $\sqrt{s_{\mathrm{NN}}}=2.76$ TeV, Phys. Lett. B770 (2017) 357-379, [1611.01510].

[16] CMS collaboration, A. M. Sirunyan et al., Measurement of nuclear modification factors of $\Upsilon(1 S)$, $\Upsilon(2 S)$, and $\Upsilon(3 S)$ mesons in PbPb collisions at $\sqrt{s_{\mathrm{NN}}}=5.02 \mathrm{TeV}, 1805.09215$.

[17] K. Kovarik et al., nCTEQ15 - Global analysis of nuclear parton distributions with uncertainties in the CTEQ framework, Phys. Rev. D93 (2016) 085037, [1509.00792];

J.-P. Lansberg and H.-S. Shao, Towards an automated tool to evaluate the impact of the nuclear modification of the gluon density on quarkonium, D and B meson production in proton-nucleus collisions, Eur. Phys. J. C77 (2017) 1, [1610.05382].

[18] K. J. Eskola, H. Paukkunen and C. A. Salgado, EPS09: A New Generation of NLO and LO Nuclear Parton Distribution Functions, JHEP 04 (2009) 065, [0 902 . 4154].

[19] LHCb collaboration, R. Aaij et al., Study of $\Upsilon$ production in pPb collisions at $\sqrt{s_{N N}}=8.16 \mathrm{TeV}$, 1810.07655. 